\title{
Sign-Changing Solutions for Discrete Dirichlet Boundary Value Problem
}

\author{
Yuhua Long, Baoling Zeng \\ School of Mathematics and Information Science, Guangzhou University, Guangzhou, China \\ Email: longyuhua214@163.com
}

How to cite this paper: Long, Y.H. and Zeng, B.L. (2017) Sign-Changing Solutions for Discrete Dirichlet Boundary Value Problem. Journal of Applied Mathematics and Physics, 5, 2228-2243.

https://doi.org/10.4236/jamp.2017.511182

Received: September 7, 2017

Accepted: November 20, 2017

Published: November 23, 2017

Copyright $\odot 2017$ by authors and Scientific Research Publishing Inc. This work is licensed under the Creative Commons Attribution International License (CC BY 4.0).

http://creativecommons.org/licenses/by/4.0/

\section{(c) (i) Open Access}

\begin{abstract}
Using invariant sets of descending flow and variational methods, we establish some sufficient conditions on the existence of sign-changing solutions, positive solutions and negative solutions for second-order nonlinear difference equations with Dirichlet boundary value problem. Some results in the literature are improved.
\end{abstract}

\section{Keywords}

Sign-Changing Solution, Difference Equation, Dirichlet Boundary Value

Problem, Invariant Sets of Descending Flow

\section{Introduction}

Let $N, Z$ and $R$ denote sets of all natural numbers, integers and real numbers, respectively. We consider the existence of sign-changing solutions, positive solutions and negative solutions for the following second-order nonlinear difference equation with Dirichlet boundary value problem (BVP for short)

$$
\left\{\begin{array}{l}
-\Delta^{2} x(k-1)=f(k, x(k)), k \in[1, T] \\
x(0)=x(T+1)=0
\end{array}\right.
$$

where $T \geq 2$ is a given integer and $[1, T]:=\{1,2, \cdots, T\}, f:[1, T] \times R \rightarrow R$ is continuous in the second variable, $\Delta$ denotes the forward difference operator defined by $\Delta x(k)=x(k+1)-x(k), \Delta^{2} x(k)=\Delta(\Delta x(k))$.

In recent years, many authors devoted to the study of (1.1) by employing various methods and obtained some interesting results. Here we mention a few. Employing critical point theory, Agarwal [1] established the existence results of multiple positive solutions. While the nonlinearity is discontinuous, Zhang [2] gained another new multiple solutions. Zhang and Sun [3] obtained two exis- 
tence results of multiple solutions. By aid of algebra and Krasnoselskii fixed point theorem, Luo [4] investigated the existence of positive solutions.

Study on the sign-changing solutions is a very important research field both in differential equations and difference equations. As to the sign-changing solutions for differential equations, many scholars achieved excellent results [5]-[14] by making using of a variety of methods and techniques, such as Leray-Schauder degree theory, fixed point index theory, topological degree theory, invariant sets of descending flow, critical point theory and etc.. Among them, invariant sets of descending flow play an important role, which was first used by Sun [10]. However, to the authors' knowledge, there are few literatures that considered sign-changing solutions for difference equations. Making use of invariant sets of descending flow, [15] studied periodic boundary value problem

$$
\left\{\begin{array}{l}
-\Delta[p(k-1) \Delta x(k-1)]+q(k) x(k)=f(k, x(k)), \quad k \in[1, T] \\
x(0)=x(T), \quad \Delta x(0)=\Delta x(T) .
\end{array}\right.
$$

In this paper, our purpose is to establish some sufficient conditions for the existence of solutions for (1.1). First, we will construct a functional $I$ such that solutions of (1.1) correspond to critical points of $I$. Then, by using invariant sets of descending flow and Mountain pass lemma, we obtain sign-changing solutions, negative solutions and positive solutions for (1.1).

\section{Preliminaries and Main Results}

Given $m \geq 0$, let $G=\{x:[0, T+1] \rightarrow R \mid x(0)=x(T+1)=0\}$ be a $T$-dimensional Hilbert space which is equipped with the inner product

$$
\langle x, y\rangle_{m}=\sum_{k=1}^{T+1} \Delta x(k-1) \Delta y(k-1)+\sum_{k=1}^{T} m x(k) y(k),
$$

then the norm $\|\cdot\|_{m}$ can be induced by

$$
\|x\|_{m}=\left(\sum_{k=1}^{T+1}|\Delta x(k-1)|^{2}+\sum_{k=1}^{T} m|x(k)|^{2}\right)^{\frac{1}{2}} .
$$

Let $H$ be the $T$-dimensional Hilbert space equipped with the usual inner product $(,$,$) and the usual norm \|\cdot\|$. It is not difficult to see that $G$ is isomorphic to $H,\|\cdot\|_{m}$ and $\|\cdot\|$ are equivalent. Denote $x^{+}=\max \{x, 0\}$, $x^{-}=\min \{x, 0\}$. Then for any $x \in H$, we find $\langle\cdot, \cdot\rangle_{m} \geq 0$.

Define functional $I: H \rightarrow R$ as

$$
I(x)=\frac{1}{2} \sum_{k=1}^{T+1}|\Delta x(k-1)|^{2}-\sum_{k=1}^{T} F(k, x(k)) .
$$

For any $x=(x(1), x(2), \cdots, x(T))^{\tau} \in H, I(x)$ can be rewritten as

$$
I(x)=\frac{1}{2}(A x, x)-\sum_{k=1}^{T} F(k, x(k)),
$$

Here $\alpha^{\tau}$ is the transpose of the vector $\alpha$ on $H$ and 


$$
A=\left(\begin{array}{cccccc}
2 & -1 & 0 & \cdots & 0 & 0 \\
-1 & 2 & -1 & \cdots & 0 & 0 \\
0 & -1 & 2 & \cdots & 0 & 0 \\
\vdots & \vdots & \vdots & \ddots & \vdots & \vdots \\
0 & 0 & 0 & \cdots & 2 & -1 \\
0 & 0 & 0 & \cdots & -1 & 2
\end{array}\right)_{T \times T}
$$

In the following, we first consider the linear eigenvalue problem corresponding to (1.1)

$$
\left\{\begin{array}{l}
-\Delta^{2} x(k-1)=\lambda x(k), k \in[1, T] \\
x(0)=x(T+1)=0
\end{array}\right.
$$

By direct computation, we get eigenvalues of (2.3) as

$$
\lambda_{k}=4 \sin ^{2} \frac{k \pi}{2(T+1)}, k=1,2, \cdots, T .
$$

Denote $\left\{z_{k}\right\}_{k=1}^{T}$ be the corresponding eigenvectors of $\left\{\lambda_{k}\right\}_{k=1}^{T}$, where

$$
z_{k}(j)=\sqrt{\frac{2}{T+1}} \sin \frac{k j \pi}{T+1}, k, j=1,2, \cdots, T .
$$

It is obvious that $0<\lambda_{k}<4, \lambda_{1}=4 \sin ^{2} \frac{\pi}{2(T+1)}>0$ and $z_{1}(j)=\sqrt{\frac{2}{T+1}} \sin \frac{\pi j}{T+1}>0$ for all $k, j \in[1, T]$. Note that $\lambda_{1}, \lambda_{2}, \cdots, \lambda_{T}$ are also eigenvalues of matrix $A$.

Next, for $m \geq 0$, we consider BVP

$$
\left\{\begin{array}{l}
-\Delta^{2} x(k-1)+m x(k)=h(k), \quad k \in[1, T] \\
x(0)=x(T+1)=0
\end{array}\right.
$$

where $h:[1, T] \rightarrow R$. It is not hard to know that (2.5) and the system of linear algebra equations $(A+m I) x=h$ are equivalent, then the unique solution of (2.5) can be expressed by

$$
x=(A+m I)^{-1} h
$$

On the other side, we have

Lemma 2.1 The unique solution of (2.5) is

$$
x(k)=\sum_{s=1}^{T} G_{m}(k, s) h(s), k \in[0, T+1],
$$

here $G_{m}(k, s)$ can be written as

$$
\begin{aligned}
& G_{m}(k, s)=\left\{\begin{array}{l}
\frac{\left(P^{s-T-1}-P^{T+1-s}\right)\left(P^{k}-P^{-k}\right)}{W}, 0 \leq k \leq s \leq T+1, \\
\frac{\left(P^{k-T-1}-P^{T+1-k}\right)\left(P^{s}-P^{-s}\right)}{W}, 0 \leq s \leq k \leq T+1,
\end{array}\right. \\
& W=\left(P^{T+1}-P^{-T-1}\right)\left(P^{-1}-P\right) .
\end{aligned}
$$


Proof. First consider the homogeneous equation of (2.5)

$$
\left\{\begin{array}{l}
-\Delta^{2} x(k-1)+m x(k)=0, \quad k \in[1, T] \\
x(0)=x(T+1)=0
\end{array}\right.
$$

then the corresponding characteristic equation of (2.7) is

$$
p^{2}-(2+m) p+1=0
$$

Since $(2+m)^{2}-4>0$, which means we have

$$
p_{1}=\frac{2+m+\sqrt{4 m+m^{2}}}{2}, \quad p_{2}=\frac{2+m-\sqrt{4 m+m^{2}}}{2} .
$$

Two independent solutions of (2.7) can be expressed by $x_{1}(k)=p_{1}^{k}$ and $x_{2}(k)=p_{2}^{k}$. Therefore, the general solution of (2.5) is $x(k)=a_{1}(k) p_{1}^{k}+a_{2}(k) p_{2}^{k}$.

The next step is to determine coefficients $a_{1}(k), a_{2}(k)$. Now using the method of variation of constant, it follows

$$
\left\{\begin{array}{l}
\Delta a_{1}(k-1) p_{1}^{k}+\Delta a_{2}(k-1) p_{2}^{k}=0 \\
\Delta a_{1}(k-1) p_{1}^{k+1}+\Delta a_{2}(k-1) p_{2}^{k+1}=-h(k) .
\end{array}\right.
$$

Then

$$
\begin{aligned}
& \Delta a_{1}(k-1)=\frac{p_{2}^{k} h(k)}{p_{2}-p_{1}} \\
& \Delta a_{2}(k-1)=-\frac{p_{1}^{k} h(k)}{p_{2}-p_{1}} .
\end{aligned}
$$

Moreover

$$
\begin{aligned}
& a_{1}(k)=a_{1}(0)+\sum_{s=1}^{k} \frac{p_{2}^{s} h(s)}{p_{2}-p_{1}}, \\
& a_{2}(k)=a_{2}(0)-\sum_{s=1}^{k} \frac{p_{1}^{s} h(s)}{p_{2}-p_{1}} .
\end{aligned}
$$

Thus, the general solution of (2.5) is

$$
x(k)=\left[a_{1}(0)+\sum_{s=1}^{k} \frac{p_{2}^{s} h(s)}{p_{2}-p_{1}}\right] p_{1}^{k}+\left[a_{2}(0)-\sum_{s=1}^{k} \frac{p_{1}^{s} h(s)}{p_{2}-p_{1}}\right] p_{2}^{k} .
$$

Using initial conditions, we find $a_{1}(0)=-a_{2}(0)$ and

$$
a_{1}(0)=\sum_{s=1}^{T} \frac{\left(p_{1}^{s} p_{2}^{T+1}-p_{2}^{s} p_{1}^{T+1}\right)}{\left(p_{1}^{T+1}-p_{2}^{T+1}\right)\left(p_{2}-p_{1}\right)} h(s)
$$

Write $W=\left(p_{1}^{T+1}-P_{2}^{T+1}\right)\left(p_{2}-p_{1}\right), \quad p_{1}=p_{2}^{-1}=P$, then

$x(k)=\frac{1}{W}\left[\sum_{s=1}^{T}\left(P^{s-T-1}-P^{T+1-s}\right)\left(P^{k}-P^{-k}\right)+\sum_{s=1}^{k}\left(P^{k-s}-P^{s-k}\right)\left(P^{T+1}-P^{-T-1}\right)\right] h(s)$.

Hence, we achieve the unique solution of (2.5)

$$
x(k)=\sum_{s=1}^{T} G_{m}(k, s) h(s), k \in[0, T+1],
$$


here $G_{m}(k, s)$ can be written as

$$
G_{m}(k, s)=\left\{\begin{array}{l}
\frac{\left(P^{s-T-1}-P^{T+1-s}\right)\left(P^{k}-P^{-k}\right)}{W}, 0 \leq k \leq s \leq T+1, \\
\frac{\left(P^{k-T-1}-P^{T+1-k}\right)\left(P^{s}-P^{-s}\right)}{W}, 0 \leq s \leq k \leq T+1 .
\end{array}\right.
$$

Remark 2.1 From Lemma 2.1, we have

$$
G_{m}(k, s)=G_{m}(s, k)>0, \quad k, s \in[1, T] .
$$

Define $K_{m}, f_{m}, A_{m}: H \rightarrow H$ as follows

$$
\begin{aligned}
& \left(K_{m} x\right)(k)=\sum_{s=1}^{T} G_{m}(k, s) x(s), x \in H, k \in[1, T] ; \\
& \left(f_{m} x\right)(k)=f(k, x(k))+m x(k), x \in H, k \in[1, T] ; \\
& A_{m}=K_{m} f_{m},
\end{aligned}
$$

where $A_{m}: H \rightarrow H$ is a completely continuous operator. Combining (2.6) with Lemma 2.1, we achieve that $K_{m}=(A+m I)^{-1}$.

Remark 2.2 According to Lemma 2.1, it is not difficult to know that $\{x(k)\}_{k=0}^{T+1}$ is a solution of (1.1) if and only if $\{x(k)\}_{k=1}^{T}$ is a fixed point of $A_{m}$.

Lemma 2.2 The functional $I$ defined by (2.1) is Frechet differentiable on $\mathrm{H}$ and $I^{\prime}(x)$ has the expression $I^{\prime}(x)=x-K_{m} f_{m} x$ for $x \in H$.

Proof. For any $x, y \in H$, using the mean value theorem, it follows

$$
\begin{aligned}
& I(x+y)-I(x) \\
& =\frac{1}{2} \sum_{k=1}^{T}|\Delta y(k-1)|^{2}+\sum_{k=1}^{T+1} \Delta x(k-1) \Delta y(k-1)-\sum_{k=1}^{T} f(k, x(k)+\theta(k) y(k)) y(k),
\end{aligned}
$$

Here $\theta(k) \in(0,1), k \in[1, T]$. As $f$ is continuous in $x$, we find

$$
\begin{aligned}
& I(x+y)-I(x)-\langle x, y\rangle_{m}+\sum_{k=1}^{T}(f(k, x(k))+m x(k)) y(k) \\
& =\sum_{k=1}^{T}[f(k, x(k))-f(k, x(k)+\theta(k) y(k))] y(k)+\frac{1}{2}\|y\|_{m}^{2}-\frac{1}{2} m\|y\|^{2} \\
& =\|y\|_{m} o(1)
\end{aligned}
$$

which leads to

$\lim _{\|y\|_{m} \rightarrow 0} \frac{1}{\|y\|_{m}}\left(\sum_{k=1}^{T}[f(k, x(k))-f(k, x(k)+\theta(k) y(k))] y(k)+\frac{1}{2}\|y\|_{m}^{2}-\frac{1}{2} m\|y\|^{2}\right)=0$

thus we can immediately conclude that $I$ is Frechet differentiable on $H$ and

$$
\left\langle I^{\prime}(x), y\right\rangle_{m}=\langle x, y\rangle_{m}-\sum_{k=1}^{T}(f(k, x(k))+m x(k)) y(k)
$$

On the other side, for all $x=\{x(k)\} \in H$ and $z=\{z(k)\} \in H$, there holds

$$
\sum_{k=1}^{T} \Delta^{2} x(k-1) z(k)=-\sum_{k=1}^{T+1} \Delta x(k-1) \Delta z(k-1) .
$$

Making use of the definition of inner product and Lemma 2.1, it follows 


$$
\begin{aligned}
& \left\langle x-K_{m} f_{m} x, y\right\rangle_{m} \\
& =\langle x, y\rangle_{m}-\sum_{k=1}^{T+1} \Delta\left(K_{m} f_{m} x\right)(k-1) \Delta y(k-1)-\sum_{k=1}^{T} m\left(K_{m} f_{m} x\right)(k) y(k) \\
& =\langle x, y\rangle_{m}-\sum_{k=1}^{T}\left\{-\Delta^{2}\left(K_{m} f_{m} x\right)(k-1)+m\left(K_{m} f_{m} x\right)(k)\right\} y(k) \\
& =\langle x, y\rangle_{m}-\sum_{k=1}^{T}(f(k, x(k))+m x(k)) y(k)
\end{aligned}
$$

Then $\left\langle I^{\prime}(x), y\right\rangle_{m}=\left\langle x-K_{m} f_{m} x, y\right\rangle_{m}$ for all $x, y \in H$, that is to say, $I^{\prime}(x)=x-K_{m} f_{m} x$.

Remark 2.3 According to Lemma 2.2 and Remark 2.2, we find that critical points of $I$ defined on $H$ are precisely solutions of (1.1).

Now, we give some necessary lemmas and definitions.

Definition 2.1 ([16]) Let $I \in C^{1}(H, R), I$ is said to be satisfied Palais-Smale condition((PS)condition for short) if every sequence $\left\{x_{n}\right\} \subset H$ such that $I\left(x_{n}\right)$ is bounded and $I^{\prime}\left(x_{n}\right) \rightarrow 0(n \rightarrow \infty)$ has a convergent subsequence in $H$.

Definition 2.2 ([17]) Assume $I \in C^{1}(H, R)$. If any sequence $\left\{x_{n}\right\}$ for which $I\left(x_{n}\right)$ is bounded and $\left(1+\left\|x_{n}\right\|_{m}\right)\left\|I^{\prime}\left(x_{n}\right)\right\|_{m} \rightarrow 0(n \rightarrow \infty)$ possesses a convergent subsequence in $H$, then we say that $I$ satisfies the Cerami condition $((\mathrm{C})$ condition for short).

Lemma 2.3 (Mountain pass lemma [16]) Let $H$ be a real Hilbert space, assume that $I \in C^{1}(H, R)$ satisfies the (PS) condition and the following conditions:

$\left(\mathrm{H}_{1}\right)$ There exist constants $\rho>0$ and $\alpha>0$ such that $I(x) \geq a$ for all $x \in \partial B_{\rho}$.

$\left(\mathrm{H}_{2}\right)$ There exists $x_{0} \notin B_{\rho}$ such that $I\left(x_{0}\right) \leq 0$.

Then $I$ has a critical value $c \geq a$, moreover, $c$ can be characterized as

$$
c=\inf _{h \in \Gamma} \max _{s \in[0,1]} I(h(s)),
$$

here

$$
\Gamma=\left\{h \in(C[0,1], H) \mid h(0)=0, h(1)=x_{0}\right\},
$$

$B_{\rho}$ be the open ball in $H$ with radius $\rho$ and centered at $0, \partial B_{\rho}$ denote boundary of $B_{\rho}$.

Lemma 2.4 ([11]) Let $H$ be a Hilbert space, there are two open convex subsets $B_{1}$ and $B_{2}$ on $H$ with $A_{m}\left(\partial B_{1}\right) \subset B_{1}, A_{m}\left(\partial B_{2}\right) \subset B_{2}$ and $B_{1} \cap B_{2} \neq \phi$. If $I \in C^{1}(H, R)$ satisfies the (PS) condition and $I^{\prime}(x)=x-A_{m} x$ for all $x \in H$. Assume there is a path $g:[1, T] \rightarrow H$ such that $g(0) \in B_{1} \backslash B_{2}, \quad g(1) \in B_{2} \backslash B_{1}$ and

$$
\inf _{x \in B_{1} \wedge \bar{B}_{2}} I(x)>\sup _{\tau \in[0,1]} I(g(\tau))
$$

then $I$ has at least four critical points, one in $H \backslash\left(\overline{B_{1}} \cup \overline{B_{2}}\right)$, one in $B_{1} \backslash \overline{B_{2}}$, one in $B_{2} \backslash \overline{B_{1}}$, and one in $B_{1} \cap B_{2}$. 
Remark 2.4 By Theorem 5.1 [17], we can replace (PS) condition by weaker (C) condition in Lemma 2.4 .

Throughout this paper, we assume that

$\left(\mathrm{J}_{1}\right) \quad f_{0}=\max _{k \in[1, T]} \limsup _{u \rightarrow 0}\left|\frac{f(k, u)}{u}\right|<\lambda_{1}$

(J $\left.\mathrm{J}_{2}\right) \lim _{|u| \rightarrow \infty} \frac{f(k, u)}{u}=r$ for $k \in[1, T]$ where $r \in(0,+\infty)$ is a constant, or $r=+\infty, v>2$ and $C>0$ satisfy

$$
|f(k, u)| \leq C\left(1+|u|^{v-1}\right) .
$$

$\left(\mathrm{J}_{3}\right)$ (i) $\lim _{|u| \rightarrow \infty}[u f(k, u)-2 F(k, u)]=-\infty, \forall k \in[1, T]$

or

(ii) $\lim _{|u| \rightarrow \infty}[u f(k, u)-2 F(k, u)]=+\infty, \forall k \in[1, T]$.

where $F(k, u)=\int_{0}^{u} f(k, s) \mathrm{d} s$.

At last, we state our main results as following.

Theorem 2.1 Suppose $\left(\mathrm{J}_{1}\right)$ and $\left(\mathrm{J}_{2}\right)$ and $r>\lambda_{2}$. Then one has the following.

(i) If $r \in\left(\lambda_{2},+\infty\right)$ is not an eigenvalue of (2.3), then (1.1) has at least three nontrivial solutions, one sign-changing, one positive and one negative.

(ii) If $r$ is an eigenvalue of (2.3) and $\left(\mathrm{J}_{3}\right)$ holds, then the conclusion of (i) is true.

Theorem 2.2 If $\liminf _{|u| \rightarrow \infty} \frac{f(k, u)}{u}>\lambda_{1}$ and $\liminf _{u \rightarrow 0} \frac{f(k, u)}{u}<\lambda_{1}$ for all $k \in[1, T]$. Then (1.1) has at least two nontrivial solutions, one negative and one positive.

From Theorem 2.2, we can get

Corollary 2.3 Suppose $f(k, 0)=0$ for any $k \in[1, T]$, we have:

(i) If $\liminf _{u \rightarrow-\infty} \frac{f(k, u)}{u}>\lambda_{1}$ and $\liminf _{u \rightarrow 0^{-}} \frac{f(k, u)}{u}<\lambda_{1}$ for any $k \in[1, T]$, then

(1.1) has at least a negative solution.

(ii) If $\liminf _{u \rightarrow+\infty} \frac{f(k, u)}{u}>\lambda_{1}$ and $\liminf _{u \rightarrow 0^{+}} \frac{f(k, u)}{u}<\lambda_{1}$ for any $k \in[1, T]$, then

(1.1) has at least a positive solution.

Our results improve previous work in the following way:

(1) [1] [2] [3] [4] considered Dirichlet boundary value problem, but it is unknown whether the solutions are sign-changing. While in this paper, the nonlinear term $f$ can change sign.

(2) The nonlinearity $f$ satisfies classical Ambrosett-Rabinowitz superlinear condition in [11] [12] [13] or locally Lipschitz continuity in [7] [8] [14], which are not used in our results.

\section{Existence of Sign-Changing Solutions of (1.1)}

In this section, we shall make use of Lemma 2.4 to complete the proof of Theo- 
rem 2.2. Let convex cones $\Lambda=\{x \in H: x \geq 0\}$ and $-\Lambda=\{x \in H: x \leq 0\}$. The distance respecting to \|\|$_{m}$ in $H$ is written by dist $_{m}$. For arbitrary $\varepsilon>0$, we denote

$$
B_{\varepsilon}^{+}=\left\{x \in H: \operatorname{dist}_{m}(x, \Lambda)<\varepsilon\right\}, \quad B_{\varepsilon}^{-}=\left\{x \in H: \operatorname{dist}_{m}(x,-\Lambda)<\varepsilon\right\}
$$

then $B_{\varepsilon}^{+}, B_{\varepsilon}^{-}$are open convex subsets on $H$ with $B_{\varepsilon}^{+} \cap B_{\varepsilon}^{-} \neq \phi$. In addition, $H \backslash\left(B_{\varepsilon}^{+} \cup B_{\varepsilon}^{-}\right)$contains only sign-changing functions.

Lemma 3.1 Suppose one of the following conditions holds.

(i) $r=+\infty$.

(ii) $r<+\infty$ is not an eigenvalue of (2.3), here $r$ is defined by $\left(\mathrm{J}_{2}\right)$.

Then the functional $I$ defined by (2.1) satisfies (PS) condition.

Proof. (i) Assume $r=+\infty$. Let $\left\{x_{n}\right\} \subset H$ be a (PS) sequence, i.e., $I\left(x_{n}\right)$ is bounded and $I^{\prime}\left(x_{n}\right) \rightarrow 0$ as $n \rightarrow \infty$. Since $H$ is a finite dimensional Hilbert space, we only need to show that $\left\{x_{n}\right\}$ is bounded. If $r=+\infty$, choosing a constant $\gamma>0$, we have $F(k, u) \geq \lambda_{T} u^{2}-\gamma$ for all $(k, u) \in[1, T] \times R$. Then

$$
\begin{aligned}
I\left(x_{n}\right) & =\frac{1}{2}\left(A x_{n}, x_{n}\right)-\sum_{k=1}^{T} F\left(k, x_{n}(k)\right) \leq \frac{1}{2} \lambda_{T}\left\|x_{n}\right\|^{2}-\lambda_{T}\left\|x_{n}\right\|^{2}+T \gamma \\
& =-\frac{1}{2} \lambda_{T}\left\|x_{n}\right\|^{2}+T \gamma
\end{aligned}
$$

furthermore,

$$
\left\|x_{n}\right\|^{2} \leq \frac{2 T \gamma-2 I\left(x_{n}\right)}{\lambda_{T}} .
$$

Since $I\left(x_{n}\right)$ is bounded, we conclude that $\left\{x_{n}\right\}$ is a bounded sequence and (PS) condition is satisfied.

(ii) suppose $r<+\infty$ is not an eigenvalue of (2.3). We are now ready to prove that $\left\{x_{n}\right\}$ is bounded. Arguing by contradiction, we suppose there is a subsequence of $\left\{x_{n}\right\}$ with $\rho_{n}=\left\|x_{n}\right\| \rightarrow+\infty(n \rightarrow+\infty)$ and for each $k \in[1, T]$, either $\left\{x_{n}(k)\right\}$ is bounded or $x_{n}(k) \rightarrow+\infty$. Put $y_{n}=\frac{x_{n}}{\rho_{n}}$. Clearly, $\left\|y_{n}\right\|=1$. Then there have a subsequence of $\left\{y_{n}\right\}$ and $y \in H$ satisfying that $y_{n} \rightarrow y$ as $n \rightarrow \infty$. Write

$$
d_{n}=\left(\frac{f\left(1, x_{n}(1)\right)}{x_{n}(1)} y_{n}(1), \frac{f\left(2, x_{n}(2)\right)}{x_{n}(2)} y_{n}(2), \cdots, \frac{f\left(T, x_{n}(T)\right)}{x_{n}(T)} y_{n}(T)\right) .
$$

Since $\lim _{|u| \rightarrow \infty} \frac{f(k, u)}{u}=r$ for all $k \in[1, T]$ and $I^{\prime}\left(x_{n}\right)=x_{n}-K_{m} f_{m} x_{n}$, we get

$$
\frac{I^{\prime}\left(x_{n}\right)}{\rho_{n}}=y_{n}-\frac{1}{\rho_{n}} K_{0} f_{0} x_{n}=y_{n}-K_{0} d_{n} \rightarrow y-K_{0} r y .
$$

Because of $\frac{I^{\prime}\left(x_{n}\right)}{\rho_{n}} \rightarrow 0$ as $n \rightarrow \infty$, we have $y-K_{0} r y \rightarrow 0$. In view of Lemma 2.2, we find that $r$ is an eigenvalue of matrix $A$, which contradicts to the assumption. So $\left\{x_{n}\right\}$ is bounded and the proof is finished. 
Lemma 3.2 I satisfies (C) condition under $\left(\mathrm{J}_{3}\right)$.

Proof . First assume $\left(\mathrm{J}_{3}\right)$ (i) be satisfied. There exists $M_{1}>0$, if $\left\{x_{n}\right\} \subset H$ be a sequence such that $I\left(x_{n}\right) \leq M_{1}$ and $\left(1+\left\|x_{n}\right\|_{m}\right)\left\|I^{\prime}\left(x_{n}\right)\right\|_{m} \leq M_{1}$, there holds

$$
\begin{aligned}
& -3 M_{1} \leq 2 I\left(x_{n}\right)-\left(1+\left\|x_{n}\right\|_{m}\right)\left\|I^{\prime}\left(x_{n}\right)\right\|_{m} \leq 2 I\left(x_{n}\right)-\left\langle I^{\prime}\left(x_{n}\right), x_{n}\right\rangle_{m} \\
& =\sum_{k=1}^{T}\left[x_{n}(k) f\left(k, x_{n}(k)\right)-2 F\left(k, x_{n}(k)\right)\right]
\end{aligned}
$$

Then we claim $\left\{x_{n}\right\}$ is bounded. Actually, if $\left\{x_{n}\right\}$ is unbounded, there possesses a subsequence of $\left\{x_{n}\right\}$ and some $k_{0} \in[1, T]$ satisfying $\left|x_{n}\left(k_{0}\right)\right| \rightarrow+\infty(n \rightarrow \infty)$. According to $\left(\mathrm{J}_{3}\right)(\mathrm{i})$, we get

$$
\begin{aligned}
& \sum_{k=1}^{T}\left[x_{n}(k) f\left(k, x_{n}(k)\right)-2 F\left(k, x_{n}(k)\right)\right] \\
& \leq\left[x_{n}\left(k_{0}\right) f\left(k, x_{n}\left(k_{0}\right)\right)-2 F\left(k, x_{n}\left(k_{0}\right)\right)\right]+(T-1) R \rightarrow-\infty
\end{aligned}
$$

and there has a positive constant $M_{2}>0$ such that $u f(k, u)-2 F(k, u) \leq M_{2}$ for any $k \in[1, T]$ and $u \in R$. Therefore,

$$
\begin{aligned}
& \sum_{k=1}^{T}\left[x_{n}(k) f\left(k, x_{n}(k)\right)-2 F\left(k, x_{n}(k)\right)\right] \\
& \leq\left[x_{n}\left(k_{0}\right) f\left(k, x_{n}\left(k_{0}\right)\right)-2 F\left(k, x_{n}\left(k_{0}\right)\right)\right]+(T-1) R \rightarrow-\infty
\end{aligned}
$$

which contradicts to (3.2). Then $I$ satisfies (C) condition.

When $\left(\mathrm{J}_{3}\right)$ (ii) holds, we can prove $I$ satisfies $(\mathrm{C})$ condition in a similar way. Then Lemma 3.2 is verified.

Lemma 3.3 If $\left(\mathrm{J}_{1}\right)$ and $\left(\mathrm{J}_{2}\right)$ hold, there exist $m \geq 0$ and $\varepsilon_{0}>0$ such that for $0<\varepsilon<\varepsilon_{0}$, we have

(i) if $x \in B_{\varepsilon}^{-}$is a nontrivial critical point of $I$ and $A_{m}\left(\partial B_{\varepsilon}^{-}\right) \subset B_{\varepsilon}^{-}$, then $x$ is a negative solution of (1.1);

(ii) if $x \in B_{\varepsilon}^{+}$is a nontrivial critical point of $I$ and $A_{m}\left(\partial B_{\varepsilon}^{+}\right) \subset B_{\varepsilon}^{+}$, then $x$ is a positive solution of (1.1).

Proof. (i) According to $\left(\mathrm{J}_{1}\right)$ and $\left(\mathrm{J}_{2}\right)$. For all $u \neq 0$ and $k \in[1, T]$, there exists $m \geq 0$ such that

$$
u(f(k, u)+m u)>0 .
$$

Let $y=A_{m}(x), x^{+}=\max \{x, 0\}, x^{-}=\max \{-x, 0\}$ for all $x \in H$. Since

$$
\|x\|_{m}^{2}=(\lambda+m)\|x\|^{2},
$$

it follows $\sqrt{\lambda_{1}+m} \leq\|x\|_{m} \leq \sqrt{\lambda_{T}+m}$ and

$$
\left\|x^{+}\right\|=\inf _{z \in-\Lambda}\|x-z\| \leq \frac{1}{\sqrt{m+\lambda_{1}}} \inf _{z \in-\Lambda}\|x-z\|_{m}=\frac{1}{\sqrt{m+\lambda_{1}}} \operatorname{dist}_{m}(x,-\Lambda) .
$$

By $\left(\mathrm{J}_{1}\right)$ and $\left(\mathrm{J}_{2}\right)$, there exist constants $\tau>0, C>0$ and $v>2$ such that

$$
|f(k, u)+m u| \leq\left(m+\lambda_{1}-\tau\right)|u|+C|u|^{v-1}, \quad \forall(k, u) \in[1, T] \times R .
$$

Choosing a positive constant $D$, since $x \in H$ is finite-dimensional, we have 


$$
|x|_{\nu}:=\left(\sum_{k=1}^{T}|x(k)|^{v}\right)^{\frac{1}{v}} \leq D \min \left\{\|x\|,\|x\|_{m}\right\}, \quad \forall x \in H .
$$

It is obviously that $|x|_{2}=\|x\|$. Moreover, $y^{+}=y-y^{-}$and $y^{-} \in-\Lambda$ imply

$$
\operatorname{dist}_{m}(y,-\Lambda) \leq\left\|y-y^{-}\right\|_{m}=\left\|y^{+}\right\| \text {. }
$$

Making use of (3.4)-(3.6), we get

$$
\begin{aligned}
\operatorname{dist}_{m}(y,-\Lambda)\left\|y^{+}\right\|_{m} & \leq\left\|y^{+}\right\|_{m}^{2} \leq\left\langle y, y^{+}\right\rangle_{m}=\left\langle A_{m} x, y^{+}\right\rangle_{m}=\left\langle K_{m} f_{m} x, y^{+}\right\rangle_{m} \\
& =\sum_{k=1}^{T}[f(k, x(k))+m x(k)] y^{+}(k) \\
& \leq \sum_{k=1}^{T}\left[f\left(k, x^{+}(k)\right)+m x^{+}(k)\right] y^{+}(k) \\
& \leq\left(m+\lambda_{1}-\tau\right)\left\|x^{+}\right\|\left\|y^{+}\right\|+C\left|x^{+}\right|_{v}^{v-1}\left|y^{+}\right|_{v} \\
& \leq\left(\frac{m+\lambda_{1}-\tau}{\sqrt{m+\lambda_{1}}}\left\|x^{+}\right\|+C D^{v}\left|x^{+}\right|^{v-1}\right)\left\|y^{+}\right\|_{m} \\
& \leq\left(\frac{m+\lambda_{1}-\tau}{m+\lambda_{1}} \operatorname{dist}_{m}(x,-\Lambda)+C_{1}\left(\operatorname{dist}_{m}(x,-\Lambda)\right)^{v-1}\right)\left\|y^{+}\right\|_{m},
\end{aligned}
$$

here $C_{1}=\frac{C D^{v}}{\sqrt{\left(m+\lambda_{1}\right)^{v-1}}}$. Hence

$$
\operatorname{dist}_{m}(y,-\Lambda) \leq \frac{m+\lambda_{1}-\tau}{m+\lambda_{1}} \operatorname{dist}_{m}(x,-\Lambda)+C_{1}\left(\operatorname{dist}_{m}(x,-\Lambda)\right)^{\nu-1} .
$$

When $C_{1}\left(\operatorname{dist}_{m}(x,-\Lambda)\right)^{\nu-2}=\frac{\tau}{2\left(m+\lambda_{1}\right)}$, there holds

Since $\frac{2\left(m+\lambda_{1}\right)-\tau}{2\left(m+\lambda_{1}\right)}<1$, we obtain

$$
A_{m}\left(\partial B_{\varepsilon}^{-}\right) \subset B_{\varepsilon}^{-}, \forall u \in B_{\varepsilon}^{-} .
$$

If $x \in B_{\varepsilon}^{-}$is a nontrivial critical point of $I$, it is clear that $I^{\prime}(x)=x-A_{m} x=0$. It follows from (3.7) that $x \in-\Lambda \backslash\{0\}$. Combining (3.3) and remark 2.1, we have $x(k)<0$. Consequently, $x$ is a negative solution of (1.1).

(ii) can be discussed similarly, we only need to change $y^{+}$to $y^{-}$to prove (ii). For simplicity, we omit its proof.

Lemma 3.4 Suppose $z_{1}, z_{2}$ be eigenvectors corresponding to eigenvalues $\lambda_{1}, \lambda_{2}$ of (2.3) and $x \in H_{2}=\operatorname{span}\left\{z_{1}, z_{2}\right\}$. If $r>\lambda_{2}$, then $I(x) \rightarrow-\infty$ as $\|x\|_{m} \rightarrow+\infty$.

Proof. (1) If $r=+\infty$. From (3.1), we can see that $I(x) \rightarrow-\infty$ as $\|x\|_{m} \rightarrow+\infty$ for any $x \in H$.

(2) Assume $r \in\left(\lambda_{2},+\infty\right)$. For $x \in H_{2}$, we have $x=\varepsilon_{1} z_{1}+\varepsilon_{2} z_{2}$. In general, we can suppose $\left(z_{1}, z_{2}\right)=0$. Thus $\|x\|^{2}=\langle x, x\rangle=\varepsilon_{1}^{2}\left\|z_{1}\right\|^{2}+\varepsilon_{2}^{2}\left\|z_{2}\right\|^{2}$ and there exists $\varepsilon$ satisfying $0<\varepsilon<\min \left\{r-\lambda_{1}, r-\lambda_{2}\right\}$. From $\lim _{|x| \rightarrow \infty} \frac{f(k, x)}{x}=r$ for any $k \in[1, T]$ 
and $x \in R$, there exists $\varsigma>0$ such that

$$
F(k, x) \geq \frac{r-\varepsilon}{2} x^{2}-\varsigma .
$$

Then for $x \in H_{2}$, it follows

$$
\begin{aligned}
I(x) & =\frac{1}{2}(A x, x)-\sum_{k=1}^{T} F(k, x(k)) \\
& \leq \frac{1}{2}\left(\lambda_{1} \varepsilon_{1}^{2}\left\|z_{1}\right\|^{2}+\lambda_{2} \varepsilon_{2}^{2}\left\|z_{2}\right\|^{2}\right)-\frac{r-\varepsilon}{2}\|x\|^{2}+T \varsigma \\
& =\frac{1}{2}\left(\lambda_{1}-r+\varepsilon\right) \varepsilon_{1}^{2}\left\|z_{1}\right\|^{2}+\frac{1}{2}\left(\lambda_{2}-r+\varepsilon\right) \varepsilon_{2}^{2}\left\|z_{2}\right\|^{2}+T \varsigma .
\end{aligned}
$$

Since $\lambda_{1}-r+\varepsilon<0$ and $\lambda_{2}-r+\varepsilon<0$, we find $I(x) \rightarrow-\infty$ as $\|x\|_{m} \rightarrow+\infty$. This completes the proof.

Now we are in the position to prove Theorem 2.1 by using Lemma 2.4 .

Proof of Theorem 2.1 From (3.5), we get

$$
F(k, x)+\frac{m}{2}|x|^{2} \leq\left(m+\lambda_{1}-\tau\right) \frac{1}{2}|x|^{2}+\frac{C}{v}|x|^{v},
$$

which combine with (3.6) gives that

$$
\begin{aligned}
I(x) & =\frac{1}{2}\langle x, x\rangle_{m}-\sum_{k=1}^{T}\left[F(k, x(k))+\frac{m}{2}|x(k)|^{2}\right] \\
& \geq \frac{1}{2}\|x\|_{m}^{2}-\frac{m+\lambda_{1}-\tau}{2}\|x\|^{2}-\frac{C}{v}|x|_{v}^{v} \\
& =\frac{\tau}{2\left(m+\lambda_{1}\right)}\|x\|_{m}^{2}-\frac{C D^{v}}{v}|x|_{m}^{v} .
\end{aligned}
$$

It follows from (3.4) that $\left\|x^{ \pm}\right\| \leq \frac{1}{\sqrt{m+\lambda_{1}}} \operatorname{dist}_{m}(x, \mp \Lambda) \leq \frac{1}{\sqrt{m+\lambda_{1}}} \varepsilon_{0}$ for any $x \in \overline{B_{\varepsilon}^{+}} \cap \overline{B_{\varepsilon}^{-}}$. Then there has $c_{0}>-\infty$ such that $\underset{u \in B_{\varepsilon}^{+} \cap \overline{B_{\varepsilon}^{-}}}{\inf } I(x)=c_{0}$. Moreover, i

view of Lemma 3.4, we can choose $R>2 \varepsilon_{0}$ such that $I(x)<c_{0}-1$ for all $x \in H_{2}$ and $\|x\|_{m}=R$. To apply Lemma 2.4, we define a path $g:[0,1] \rightarrow H_{2}$ as

$$
g(s)=R \frac{z_{1} \cos (\pi s)+z_{2} \sin (\pi s)}{\left\|z_{1} \cos (\pi s)+z_{2} \sin (\pi s)\right\|_{m}} .
$$

By direct computation, we get

$$
g(0)=R \frac{z_{1}}{\left\|z_{1}\right\|_{m}} \in B_{\varepsilon}^{+} \backslash B_{\varepsilon}^{-}, \quad g(1)=-R \frac{z_{1}}{\left\|z_{1}\right\|_{m}} \in B_{\varepsilon}^{-} \backslash B_{\varepsilon}^{+}
$$

and

$$
\underline{\inf }_{x \in B_{\varepsilon}^{+} \cap \overline{B_{\varepsilon}^{-}}} I(x)>\sup _{\tau \in[0,1]} I(g(\tau)) .
$$

Combining Lemmas 3.1, 3.3 and 2.4, we find there has a critical point in $H \backslash\left(\overline{B_{\varepsilon}^{+}} \cup \overline{B_{\varepsilon}^{-}}\right)$corresponding to a sign-changing solution of (1.1). Moreover, 
we also have a critical point in $B_{\varepsilon}^{+} \backslash \overline{B_{\varepsilon}^{-}}\left(B_{\varepsilon}^{-} \backslash \overline{B_{\varepsilon}^{+}}\right)$corresponding to a positive solution (a negative solution) of (1.1). The proof of (i) is completed.

Notice Lemma 3.2 and Remark 2.4, the proof of (ii) is analogous to (i) and we omit it.

\section{Existence of Positive Solutions of (1.1)}

In this section, we are now ready to prove existence of positive solutions of (1.1) using Lemma 2.3. Denote $x^{+}=\max \{x, 0\}$ and $x^{-}=\min \{x, 0\}$. Assume $f(k, 0)=0$ for all $k \in[1, T]$. To prove Theorem 1.2, we consider functionals

$$
I_{ \pm}(x)=\frac{1}{2}\langle x, x\rangle_{0}-\sum_{k=1}^{T} F\left(k, x^{ \pm}\right), \quad \forall x \in H .
$$

It is easy to find that critical points of the function $I_{+}\left(I_{-}\right)$correspond to positive solutions (negative solutions) of (1.1).

Lemma 4.1 If $\lim _{|u| \rightarrow \infty} \inf \frac{f(k, u)}{u}>\lambda_{1}$ for all $k \in[1, T]$, then $I_{+}$and $I_{-}$ satisfy (PS) condition.

Proof. Suppose $\left\{x_{n}\right\} \subset H$ be a sequence with $I_{+}\left(x_{n}\right)$ is bounded and $I_{+}^{\prime}\left(x_{n}\right) \rightarrow 0$ as $n \rightarrow+\infty$. Denote $\left(f_{+} x\right)(k)=f\left(k, x^{+}(k)\right)$ for $k \in[1, T]$ and $x \in H$. In view of (2.6) and $x^{-}=\min \{x, 0\} \leq 0$, there holds

$$
\left\|x_{n}^{-}\right\|_{0}^{2} \leq\left\langle x_{n}, x_{n}^{-}\right\rangle_{0} \leq\left\langle x_{n}-K f_{+} x_{n}, x_{n}^{-}\right\rangle_{0}=\left\langle I_{+}^{\prime}\left(x_{n}\right), x_{n}^{-}\right\rangle_{0}=o(1)\left\|x_{n}^{-}\right\|_{0},
$$

thus $x_{n}^{-} \rightarrow 0$ as $n \rightarrow \infty$. So we claim $\left\{x_{n}^{+}\right\}$is bounded. We assume, by contradiction, that there has a subsequence of $\left\{x_{n}\right\}$ with $\rho_{n}=\left\|x_{n}^{+}\right\|_{0} \rightarrow+\infty$ as $n \rightarrow \infty$. For each $k \in[1, T]$, either $\left\{x_{n}^{+}\right\}$is bounded or $x_{n}^{+}(k) \rightarrow+\infty$. Put $y_{n}=\frac{x_{n}^{+}}{\rho_{n}}$. then $\left\|y_{n}\right\|_{0}=1$. Moreover, there has a subsequence of $\left\{y_{n}\right\}$ and $y \in E$ satisfying $y_{n} \rightarrow y$ as $n \rightarrow \infty$.

Denoting $z_{1}>0$, the eigenvector associated with $\lambda_{1}$, we obtain

$$
\begin{aligned}
\lambda_{1} \sum_{k=1}^{T} x_{n}(k) z_{1}(k) & =-\sum_{k=1}^{T} \Delta^{2} x_{n}(k-1) z_{1}(k)=\sum_{k=1}^{T+1} \Delta x_{n}(k-1) z_{1}(k-1) \\
& =\left\langle x_{n} z_{1}\right\rangle_{0}=\left\langle K f_{+} x_{n}, z_{1}\right\rangle_{0}+I_{+}^{\prime}\left(x_{n}\right) z_{1} \\
& =\sum_{k=1}^{T} f\left(k, x_{n}^{+}(k)\right) z_{1}+\left\langle I_{+}^{\prime}\left(x_{n}\right), z_{1}\right\rangle>0 .
\end{aligned}
$$

Dividing by $\rho_{n}$, it follows immediately that

$$
\lambda_{1} \sum_{k=1}^{T} y_{n}(k) z_{1}(k)=\sum_{k=1}^{T} \frac{f\left(k, x_{n}^{+}(k)\right)}{x_{n}^{+}(k)} y_{n}(k) z_{1}(k)+o(1) .
$$

Since $\min _{k \in[1, T]|u| \rightarrow \infty} \operatorname{limf}_{n} \frac{f(k, u)}{u}>\lambda_{1}$ and $\left\|y_{n}\right\|_{0}=1$, then passing to the limit in (4.1), we get a contradiction. Hence, our claim is true. Since $H$ is finite dimensional, the above argument means that $\left\{x_{n}\right\}$ has a convergent subsequence. 
Consequently, $I_{+}$satisfies (PS) condition.

Similarly, it is not difficult to know that $I_{-}$satisfies (PS) condition. Lemma 4.1 is proved.

Proof of Theorem 2.2 From $\max _{k \in[1, T] u \rightarrow 0} \frac{f(k, u)}{u}<\lambda_{1}$, there exist $\eta>0$ and $\delta>0$ such that

$$
F(k, u)=\int_{0}^{u} f(k, s) \mathrm{d} s \leq \frac{1}{2}\left(\lambda_{1}-\eta\right)|u|^{2}, \quad k \in[1, T],|u| \leq \delta .
$$

Now if we denote $B_{\delta}=\left\{x \in H:\|x\|_{0}<\delta\right\}$, then for $x \in \partial B_{\delta}$, there holds

$$
\begin{aligned}
I_{+}(x) & =\frac{1}{2}\langle x, x\rangle_{0}-\sum_{k=1}^{T} F\left(k, x^{+}(k)\right) \geq \frac{1}{2}\|x\|_{0}^{2}-\frac{1}{2}\left(\lambda_{1}-\eta\right)\|x\|^{2} \\
& \geq \frac{1}{2}\|x\|_{0}^{2}-\frac{\lambda_{1}-\eta}{2 \lambda_{1}}\|x\|_{0}^{2}=\frac{1}{2 \lambda_{1}} \eta \delta^{2} .
\end{aligned}
$$

Because of $\min _{k \in[1, T] u \rightarrow \infty} \lim \inf \frac{f(k, u)}{u}>\lambda_{1}$, there exists a constant $\xi>0$ such that

$$
\min _{k \in[1, T] u \rightarrow \infty} \liminf _{u \rightarrow \infty} \frac{f(k, u)}{u}>\lambda_{1}+\xi
$$

Then we can choose a positive constant $C_{2}$ such that $F(k, u)=\frac{1}{2}\left(\lambda_{1}+\xi\right) u^{2}-C_{2}$ for all $(k, u) \in[1, T] \times R$. If $v$ is sufficiently large, we obtain

$$
I_{+}\left(v z_{1}\right) \leq \frac{v^{2}}{2}\left\|z_{1}\right\|_{0}^{2}-\frac{v^{2}}{2 \lambda_{1}}\left(\lambda_{1}+\xi\right)\left\|z_{1}\right\|_{0}^{2}+C_{2}=-\frac{v^{2} \xi}{2 \lambda_{1}}\left\|z_{1}\right\|_{0}^{2}+C_{2}<0 .
$$

In view of Lemma 2.3 and 4.1, we yield that there exists $\mu \in H$ such that $I_{+}^{\prime}(\mu)=0$ and $I_{+}(\mu) \geq \frac{1}{2 \lambda_{1}} \eta \delta^{2}>0$. Hence

$$
\left\|\mu^{-}\right\|_{0}^{2} \leq\left\langle\mu, \mu^{-}\right\rangle_{0} \leq\left\langle\mu-K f_{+} \mu, \mu^{-}\right\rangle_{0}=\left\langle I_{+}^{\prime}(\mu), \mu^{-}\right\rangle_{0} .
$$

Consequently, $\mu^{-}=0$. Thus $\mu=\mu^{+} \geq 0$.

If $\mu(k)=0$ for some $k \in[1, T]$, we find

$$
-\mu(k+1)-\mu(k-1)=-\Delta^{2} \mu(k-1)=f(k, u(k))=0,
$$

then $\mu(k \pm 1)=0$. If $\mu(k)=0$ somewhere in $k \in[1, T]$, it vanishes identically. By $I_{+}^{\prime}(\mu) \geq \rho>0$, we obtain $\mu(k)>0$ for $k \in[1, T]$. Therefore, $\mu$ is a positive solution of (1.1).

In a similar way as above, if we consider the case of $I_{-}$, a negative solution can be obtained. Then the proof of Theorem 2.2 is finished.

\section{Applications}

To illustrate Theorem 2.1 and Theorem 2.2, we will give two examples.

Example 5.1 Consider BVP 


$$
\left\{\begin{array}{l}
-\Delta^{2} x(k-1)=\frac{|x(k)|-n}{|x(k)|+1} m x(k), k \in[1, T] \\
x(0)=x(T+1)=0
\end{array}\right.
$$

where $m>4 \sin ^{2} \frac{\pi}{T+1}, 0<n<\frac{4 \sin ^{2} \frac{\pi}{2(T+1)}}{m}$.

By direct calculation, we get

$$
F(k, x)= \begin{cases}\frac{m x^{2}}{2}-m(n+1)(x-\ln (1+x)), & x \geq 0, \\ \frac{m x^{2}}{2}+m(n+1)(x+\ln (1-x)), & x<0 .\end{cases}
$$

and $\lim _{|x| \rightarrow \infty}[x f(k, x)-2 F(k, x)]=+\infty$ for all $k \in[1, T]$. According to (2.4), we obtain

$$
\lambda_{1}=4 \sin ^{2} \frac{\pi}{2(T+1)}, \lambda_{2}=4 \sin ^{2} \frac{\pi}{T+1} .
$$

In addition, $f_{0}=\max _{k \in[1, T]} \limsup _{x \rightarrow 0}\left|\frac{f(k, x)}{x}\right|=m n<\lambda_{1}$ and $\lim _{|x| \rightarrow \infty} \frac{f(k, x)}{x}=r=m>\lambda_{2}$. From above argument, we find all conditions of Theorem 2.1 are satisfied, thus (5.1) has at least a sign-changing solution, a positive solution and a negative solution.

For a certain case, fix $T=2$, here $m>4 \sin ^{2} \frac{\pi}{3}=3,0<n<\frac{1}{m}$, then we can choose $m=4, n=\frac{1}{5}$. After not very complicated calculation, we find

$$
\left(0, \frac{3}{5}, \frac{3}{5}, 0\right),\left(0,-\frac{3}{5},-\frac{3}{5}, 0\right),\left(0, \frac{19}{5},-\frac{19}{5}, 0\right),\left(0,-\frac{19}{5}, \frac{19}{5}, 0\right)
$$

are positive solution, sign-changing solution, sign-changing solution and negative solution of (5.1), respectively.

Remark 5.1 From above example, we can get at least three nontrivial solutions of (1.1), one sign-changing, one positive and one negative if the nonlinearity $f$ satisfy all the conditions of Theorem 2.1.

Example 5.2 Consider BVP

$$
\left\{\begin{array}{l}
-\Delta^{2} x(k-1)=x^{3}(k)+a x(k), k \in[1, T] \\
x(0)=x(T+1)=0
\end{array}\right.
$$

here $a<4 \sin ^{2} \frac{\pi}{2(T+1)}$.

From (2.4), it is easy to see that $\lambda_{1}=4 \sin ^{2} \frac{\pi}{2(T+1)}$. Moreover, $f(k, 0)=0$, $\limsup _{x \rightarrow 0} \frac{f(k, x)}{x}=a<\lambda_{1}$ and $\limsup _{|x| \rightarrow \infty} \frac{f(k, x)}{x} \geq \lambda_{1}$ for all $k \in[1, T]$. Therefore, 
it follows from Theorem 2.2 that (5.2) has at least a positive solution and a negative solution.

In the case of $T=2$, because of $a<4 \sin ^{2} \frac{\pi}{6}=1$, we can choose $a=\frac{1}{2}$. After direct computation, we get that $\left(0, \frac{\sqrt{2}}{2}, \frac{\sqrt{2}}{2}, 0\right)$ and $\left(0,-\frac{\sqrt{2}}{2},-\frac{\sqrt{2}}{2}, 0\right)$ are positive solution and negative solution of (5.2), respectively.

Remark 5.2 From example 5.2, it is not difficult to know that if the nonlinearity $f$ satisfy all the conditions of Theorem 2.2 , we can obtain at least a positive solution and a negative solution of (1.1).

\section{Conclusion}

In this manuscript, some sufficient conditions on the existence of sign-changing solutions, positive solutions and negative solutions for a class of second-order nonlinear difference equations were established with Dirichlet boundary value problem by using invariant sets of descending flow and variational methods. Our results improve some existed ones in some literatures, because we not only establish some sufficient conditions on the existence of sign-changing solutions, but also we allow the nonlinearity $f$ to dissatisfy Ambrosett-Rabinowitz type condition or locally Lipschitz continuity and to change sign.

\section{Acknowledgements}

This work was supported by Key Laboratory of Mathematics and Interdisciplinary Sciences of Guangdong Higher Education Institute. The authors would like to thank the reviewer for the valuable comments and suggestions, thanks.

\section{References}

[1] Agarwal, R.P., Perera, K. and O’Regan, D. (2004) Multiple Positive Solutions of Singular and Nonsingular Discrete Problems via Variational Methods. NNonlinear Analysis: Theory, Methods \& Applications, 58, 69-73. https://doi.org/10.1016/j.na.2003.11.012

[2] Zhang, G.Q., Zhang, W.G. and Liu, S.Y. (2007) Multiplicity Result for a Discrete Eigenvalue Problem with Discontinuous Nonlinearities. Journal of Mathematical Analysis and Applications, 328, 1068-1074. https://doi.org/10.1016/j.jmaa.2006.05.077

[3] Zhang, G.D. and Sun, H.R. (2010) Existence of Multipe Solutions for a Class of Boundary Value Problem of Second Order Difference Equation. Journal of Northwest Normal University, 46, 11-14.

[4] Luo, L.J. (2004) Existence of Positive Solutions to Second-Order Difference Equations Boundary Value Problem. Journal of Guangzhou University, 3, 501-503. (in Chinese).

[5] Xu, X.A. (2004) Multiple Sign-Changing Solutions for Some M-Point Boundary Value Problem. Electronic Journal of Differential Equations, 89, 281-286.

[6] Li, F.Y., Liang, Z.P., Zhang, Q. and Li, Y.H. (2007) On Sign-Changing Solutions for Nonlinear Operator Equations. Journal of Mathematical Analysis and Applications, 
327, 1010-1028.

[7] Zhang, K.M. and Xie, X.J. (2009) Existence of Sign-Changing Solutions for Some Asymptotically Linear Three-Point Boundary Value Problems. Nonlinear Analysis, 70, 2796-2805.

[8] Zhang, Z.T. and Perera, K. (2006) Sign-Changing Solutions of Kirchhoff Type Problems via Invariant Sets of Descending Flow. Journal of Mathematical Analysis and Applications, 317, 456-463.

[9] Mao, A.M. and Zhang, Z.T. (2009) Sign-Changing and Multiple Solutions of Kirchhoff Type Problems. Nonlinear Analysis, 70, 1275-1287.

[10] Sun, J.X. (1984) On Some Problems about Nonlinear Operators. PhD Thesis, Shandong University, Jinan.

[11] Liu, Z.L. and Sun, J.X. (2001) Invariant Sets of Descending Flow in Critical Point Theory with Applications to Nonlinear Differential Equations. Journal of Differential Equations, 172, 257-299. https://doi.org/10.1006/jdeq.2000.3867

[12] Bartsch, T. and Liu, Z.L. (2004) On a Superlinear Elliptic p-Laplacian Equation. Journal of Differential Equations, 198, 149-175.

[13] Bartsch, T., Liu, Z.L. and Weth, T. (2005) Nodal Solutions of a p-Laplacian Equation. Proceedings of the London Mathematical Society, 91, 129-152.

https://doi.org/10.1112/S0024611504015187

[14] Dancer, E.N. and Zhang, Z.T. (2000) Fucik Spectrum, Sign-Changing and Multiple Solutions for Semilinear Elliptic Boundary Value Problems with Resonance at Infinity. Journal of Mathematical Analysis and Applications, 250, 449-464.

https://doi.org/10.1006/jmaa.2000.6969

[15] He, T.S., Zhou, Y.W., Xu, Y.T. and Chen, C.Y. (2015) Sign-Changing Solutions for Discrete Second-Order Periodic Boundary Value Problems. Bulletin of the Malaysian Mathematical Sciences Society, 38, 181-195. https://doi.org/10.1007/s40840-014-0012-1

[16] Rabinowitz, P.H. (1986) Minimax Methods in Critical Point Theory with Applications to Differential Equations. CBMS Regional Conference Series in Mathematics, American Mathematical Society, Providence, Vol. 65.

[17] Liu, X.Q. and Liu, J.Q. (2011) On a Boundary Value Problem in the Half-Space. Journal of Differential Equations, 250, 2099-2142. 